\title{
Research Article \\ Epinephrine Use in Endoscopic Ear Surgery: \\ Quantitative Safety Assessment
}

Matteo Alicandri-Ciufelli MD ${ }^{1}$, Giulia Molinari $\mathrm{MD}^{1 *}$, Sven Beckmann MD², Marco Caversaccio MD², Livio Presutti MD ${ }^{1}$, Lukas Anschuetz $\mathrm{MD}^{2}$

${ }^{1}$ Department of Otorhinolaryngology - Head \& Neck Surgery, AOU Policlinico di Modena, University of Modena and Reggio Emilia, Modena, Italy

${ }^{2}$ Department of Otorhinolaryngology - Head and Neck Surgery, Inselspital, Bern University Hospital, University of Bern, Bern, Switzerland

Short Title: Epinephrine Use in Endoscopic Ear Surgery

*Corresponding Author

Giulia Molinari, MD

Department of Otorhinolaryngology - Head \& Neck Surgery, AOU Policlinico di Modena, University of Modena and Reggio Emilia

Largo del Pozzo, 71

41125 Modena (MO), Italy

Tel: +390594222402

Fax: +390594224527

E-mail: dr.giuliamolinari@gmail.com

Keywords: endoscopic ear surgery; bleeding; epinephrine; safety; efficacy 


\section{Abstract}

Introduction: The management of bleeding in exclusive endoscopic ear surgery (EES) is largely dependent on epinephrine use. However, to date its suitability and safety has not been assessed. The aim of the present study would be to assess the safety profile of topical application and/or local infiltration of diluted epinephrine during EES regarding the intra- and postoperative periods. We hypothesize that epinephrine may be safely used during EES.

Methods: Retrospective analysis of 90 EES cases performed at the University Hospital of Modena, Italy. Patient's charts and video recordings of the operations were assessed.

Results: Epinephrine was used in all cases for hemostatic purposes as following: (1) diluted epinephrine (1:200.000) injection: mean $1.2 \mathrm{~mL}$ (range: 0 - 3.5) corresponding to $0.006 \mathrm{mg}$ (range 0 0.018 ) and (2) topical application (1:1000) directly in the surgical field: mean 7 cottonoids (range: 0 18) corresponding to mean $0.56 \mathrm{mg}$ (range: 0 -1.44). No major cardiovascular adverse effects were assessed. Only 2 intraoperative hypertensive events were recorded. One sensorineural hearing loss was observed in a case of cholesteatoma involving the stapes; no facial palsy occurred during the study period.

Conclusion: The combined use of local injection and topical epinephrine is a safe and effective strategy to control bleeding in exclusive EES, when used within the dilutions and quantities reported in this study. 


\section{Introduction}

Endoscopic ear surgery (EES) is gaining popularity worldwide for the treatment of a variety of conditions, from cholesteatoma of the middle ear to pathologies of the lateral skull base. One of the most significant advantages of the endoscopic technique is the direct and clear visualization of anatomical structures [1]. However, the endoscope is located directly in the operating field, where it can easily be soiled, causing a deterioration of the surgical view. As EES is largely a one-handed technique, it is not possible to use the surgical instruments simultaneously to the suction as in the microscopic technique. Moreover, the external auditory canal (EAC) and the tympanic cavity constitute a very small and delicate surgical field, where even small amounts of blood may seriously impair the quality of vision, threaten the continuation of surgery or force the surgeon to switch to an open approach. It has been shown that uncontrolled bleeding during other endoscopic procedures correlate with a higher rate of complications and prolonged operative time $[2,3]$.

Currently, this topic has not been investigated for EES. A descriptive study on the management of bleeding during EES has been recently published, showing reasonable bleeding control through widely available hemostatic agents [4]. One of the most commonly applied measures to maintain a bloodless surgical field is the application of epinephrine, a non-selective alpha-agonist vasoconstrictor. Epinephrine may prevent or control bleeding in two parallel ways during EES: it can be injected into the EAC skin prior to the elevation of the tympanomeatal flap, and epinephrinesoaked cottonoid patties can be used to pack off areas of bleeding and, at the same time, absorb the excess of blood [5].

Epinephrine-related side effects have been reported in the literature [6-8], most of them are the results of its intravascular administration. Among these, blood pressure changes (temporary hypotension or hypertension crisis), tachycardia or cardiac arrhythmia should be mentioned. Furthermore, there is some concern about the possible masking of arterial bleeding by epinephrine, which could induce post-operative bleeding, when the vasoconstrictive effect has disappeared [9]. Studies on endoscopic sinus surgery have revealed that use of topical epinephrine has some minimal systemic vascular effects and generally does not correlate with an increased risk of epistaxis [7, 9-11]. In this context, during EES, too strong vasoconstriction inside the middle ear could lead to local complications, such as facial nerve palsy or sensorineural hearing loss (SNHL).

The aim of the present study is to assess the safety profile of topical application and/or local infiltration of diluted epinephrine during EES regarding the intra- and postoperative periods. We hypothesize that epinephrine may be safely used during EES. 


\section{Materials and Methods}

A total of 90 consecutive cases of exclusive, transcanal EES for middle ear pathologies performed at the University Hospital of Modena were retrospectively analyzed. Data from patient's charts, surgical and anesthesiology reports were collected. In particular, information regarding patient age, gender, ear pathology, comorbidities and personal medications were retrieved. General surgical details (surgical time, type of anesthesia, blood pressure and heart rate) were collected and analyzed. The volume in $\mathrm{mL}$ of injected epinephrine (1:200.000) contained in $2 \%$ carbocaine was recorded. The number of epinephrine (1:1000)-soaked cottonoids used during each procedure in the EAC and the middle ear was assessed from the study of the surgical videos. The absolute values in $\mathrm{mg}$ epinephrine injected and topically applied were calculated. Moreover, the blood pressure and mean arterial pressure (MAP) were assessed throughout the intervention.

To assess the safety profile of epinephrine, both intraoperative and post-operative possible adverse reactions were taken into consideration, according to a set of basic criteria for causality assessment (temporality, strength, biologic gradient and plausibility), according to a previously published study in endoscopic sinus surgery [8].

Facial nerve paralysis and SNHL were included as adverse reactions to epinephrine only in cases where they could not be reasonably attributable to the surgical procedure itself. Intraoperative or postoperative cardiac events were defined as the necessity for additional electrocardiographic monitoring, further cardiac evaluation from a cardiologist, or prolonged hospital stay. Post-operative hemorrhage from the surgical site was also considered as a possible complication. Statistical analysis was performed with GraphPad Prism 7 (GraphPad Software, Inc, La Jolla, California).

\section{Results}

\section{Patients}

A total of 90 patients were enrolled in the present study ( 51 males and 39 females). The patient's characteristics including the performed surgeries are summarized in Table 1. The mean surgical time was 97.43 minutes; the mean hospital stay was 1,61 days.

Regarding blood thinning medications, anticoagulation was used in 1 patient and stopped 1 day before surgery, whereas anti-aggregation with acetylsalicylic acid was used in 3 patients and stopped 7 days before, ticlopidin was used in 1 patient and stopped 5 days before surgery.

Intraoperative assessment 
Epinephrine was used in all cases for hemostatic purposes as following: (1) diluted epinephrine (1:200.000) injection into the EAC skin: mean $1.2 \mathrm{~mL}$ (range: 0 - 3.5) corresponding to $0.006 \mathrm{mg}$ (range 0 - 0.018) and (2) topical application (1:1000) directly in the surgical field (EAC and middle ear): mean 7 cottonoids (range: 0 - 18) corresponding to mean $0.56 \mathrm{mg}$ (range: 0 - 1.44).

Intraoperative blood pressure peak values were recorded as: mean systolic blood pressure 94.04 $\mathrm{mmHg}$ (range: 52-155), mean diastolic blood pressure $57.34 \mathrm{mmHg}$ (range: $32-95$ ) and mean MAP $69.57 \mathrm{mmHg}$ (41-115). Mean heart rate was assessed as 66.3 beats per minute (range: $45-126)$. Mean MAP values were correlated to injected (Figure 1A) and topically applied epinephrine (Figure 1B). No statistically significant effect of epinephrine on blood pressure was observed. Similarly, no correlation of injected or applied epinephrine on heart rates was observed.

The comparison of epinephrine used and type of intervention revealed higher values for cholesteatoma surgery and tumor removal compared to stapedotomies (Figure 2). The highest mean MAP however did not differ between the interventions.

\section{Complications}

Two cases of intraoperative high blood pressure were recorded; otherwise no other intraoperative complications were documented (Table 2). Postoperative complications included one SNHL in a patient after cholesteatoma removal. In this case, the cholesteatoma involved the stapes and its removal most probably caused the SNHL. Otherwise no systemic complications or cardiovascular events were described. Two local bleedings occurred during the hospital stay, none of them required a revision surgery. No intraoperative conversion to open approaches due to excessive bleeding was necessary.

\section{Discussion}

This study assesses the safety of injected and topically applied epinephrine during exclusive, transcanal endoscopic middle ear surgery. We observed no systemic or local adverse effects related to epinephrine. Moreover, all surgeries could be safely performed using an exclusively endoscopic technique.

Literature on epinephrine use in ear surgery is scarce. Investigations regarding functional endoscopic sinus surgery (FESS) showed that local injection of epinephrine lead to significant changes of hemodynamic parameters directly after infiltration as compared to topical epinephrine [10]. Contradictorily to this finding, even systemic hypotension after local injection with epinephrine alone was reported [10]. Intraoperative hemodynamic monitoring during the use of topical epinephrine in 
FESS showed no significant changes in the majority of patients, even though a transient elevation in blood pressure was found in a subset of patients [6]. Possible cardiovascular alterations are therefore less likely to be provoked by the topical use, than by the injection of epinephrine $[8,10]$. Our data suggests no correlations regarding the quantity of epinephrine used and MAP or heart frequency. Moreover, we observed no cardiovascular events in our cohort with only two cases of intraoperative high blood pressure. These findings indicate, that epinephrine used during ear surgery may be considered safe from a cardiovascular point of view. However, we have to consider that possible toxic side effects of epinephrine such as hypertensive crisis, pulmonary edema, cardiogenic shock or even cardiac arrest are reported [12]. The causing mechanism has not yet been conclusively clarified, but epinephrine plasma levels after topical application are also elevated $[13,14]$. Therefore, it is advisable to apply epinephrine with utmost care during EES.

In our opinion, to prevent such side effects the applied concentration of epinephrine may play a crucial role. We used a dilution of 1:200.000 regarding injected epinephrine, which has a similar effect on vasoconstriction in the EAC compared to higher epinephrine concentrations [15]. Even lower concentrations (1:400.000) were shown to have a similar hemostatic effect in head and neck surgery but were not studied in ear surgery to date [16]. For topical application we used epinephrine soaked cottonoids in a dilution of 1:1000 similarly to the concentration used in FESS [10]. Optimal topical epinephrine concentration in FESS was evaluated as 1:2000 with shorter operation time and less bleeding compared to 1:10.000 and 1:50.000. However, plasma epinephrine levels were higher with less dilution and elevated intraoperative blood pressure occurred more often compared to the lower concentrations [17]. Even if we did not observe significant adverse effects of epinephrine use in EES, lower topical and injected epinephrine concentrations should be further investigated in EES. This may have important future implications as life expectancy and corresponding cardiovascular comorbidities are increasing and possible adverse effects may be more frequent. In our opinion intraoperative patient's safety is of utmost importance.

Regarding local complications due to application of epinephrine in the middle ear, we observed one case of SNHL. In this patient, the cholesteatoma eroded the stapes and its cleaning was surgically challenging. Therefore, the causative event for SNHL in this case is difficult to conclusively determine. Nonetheless an effect of topical applied epinephrine has to be considered, as topical epinephrine applied to the round window membrane can cause a transient reduction of $60 \%$ in cochlear blood flow [18]. However, in this study no postoperative SNHL as compared to preoperative measurements was observed [18]. Moreover, together with Propofol-induced hypotension, topical epinephrine is thought to elevate the auditory brainstem response threshold during the application time [19]. In 
conclusion, topical epinephrine may cause local impaired hemodynamics and insufficient autoregulation of cochlear blood flow, eventually leading to SNHL. One solution for this problem would be the use of cottonoids without epinephrine at proximity of the inner ear, especially the round and oval windows.

No facial nerve palsy was observed in our cohort. However, it has been shown, that topical application of local anesthetics with epinephrine on the facial nerve reduces its blood flow and elevates the threshold for evoked muscle action potentials in a dose and time dependent manner [20]. Despite these observations, the facial nerve function was fully restored after a certain time. We have to emphasize that topical application of adrenaline on a denudated facial nerve should be avoided in any circumstances.

In conclusion, data from the present study showed that the combined use of local injection and topical epinephrine is a safe and effective strategy to control bleeding in exclusive EES, when used within the dilutions and quantities reported in this study. 


\section{Statements}

\section{Statement of Ethics}

All procedures performed in this study involving human participants were in accordance with the ethical standards of the institutional, regional and national research committee and with the 1964 Declaration of Helsinki and its later amendments or comparable ethical standards.

Formal consent to participate to the study is not required for this type of study.

Our institutional review board does not require formal approval for this type of study.

\section{Disclosure Statement}

The authors have no conflicts of interest to declare.

\section{Funding Sources}

There was no funding source for this study. 


\section{Author Contributions}

Matteo Alicandri-Ciufelli: Conception and design, data analysis supervision. Revision of the manuscript and approval of final version. Is accountable for all aspects of the work.

Giulia Molinari: Conception and design, data collection, manuscript draft and revision of the manuscript and approval of final version. Is accountable for all aspects of the work.

Sven Beckmann: Data analysis. Manuscript draft. Revision of the manuscript and approval of final version. Is accountable for all aspects of the work.

Marco Caversaccio: Conception and design, critical senior supervision of the study. Revision of the manuscript and approval of final version. Is accountable for all aspects of the work.

Livio Presutti: Conception and design, critical senior supervision of the study. Revision of the manuscript and approval of final version. Is accountable for all aspects of the work.

Lukas Anschuetz: Conception and design, data collection, statistical analysis, manuscript draft and approval of final version. Is accountable for all aspects of the work. 


\section{References}

1. Anschuetz L, Huwendiek S, Stricker D, Yacoub A, Wimmer W, Caversaccio M. Assessment of Middle Ear Anatomy Teaching Methodologies Using Microscopy versus Endoscopy: A Randomized Comparative Study. Anat Sci Educ. 2018 [in press]

2. Qiao H, Chen J, Li W, Shen X. Intranasal atomised dexmedetomidine optimises surgical field visualisation with decreased blood loss during endoscopic sinus surgery: a randomized study. Rhinology. 2016;54(1):38-44.

3. Wormald PJ, van Renen G, Perks J, Jones JA, Langton-Hewer CD. The effect of the total intravenous anesthesia compared with inhalational anesthesia on the surgical field during endoscopic sinus surgery. Am J Rhinol. 2005 Sep-Oct;19(5):514-20.

4. Anschuetz L, Bonali M, Guarino P, Fabbri FB, Alicandri-Ciufelli M, Villari D, et al. Management of Bleeding in Exclusive Endoscopic Ear Surgery: Pilot Clinical Experience. Otolaryngol Neck Surg. 2017;157(4):700-6.

5. Presutti L, Marchioni D. Endoscopic Ear Surgery. Presutti L, Marchioni D, editors. Thieme; 2015.

6. Yim MT, Ahmed OG, Taxkashima M. Evaluating real-time effects of topical 1:1000 epinephrine in endoscopic sinus and skull-base surgery on hemodynamic parameters through intraoperative arterial line monitoring. Int Forum Allergy Rhinol. 2017;7(11):1065-9.

7. Lee TJ, Huang CC, Chang PH, Chang CJ, Chen YW. Hemostasis during functional endoscopic sinus surgery: The effect of local infiltration with adrenaline. Otolaryngol - Head Neck Surg. 2009;140(2):209-14.

8. Higgins TS, Hwang PH, Kingdom TT, Orlandi RR, Stammberger H, Han JK. Systematic review of topical vasoconstrictors in endoscopic sinus surgery. Laryngoscope. 2011;121(2):422-32.

9. Korkmaz H, Yao WC, Korkmaz M, Bleier BS. Safety and efficacy of concentrated topical epinephrine use in endoscopic endonasal surgery. Int Forum Allergy Rhinol. 2015;5(12):111823.

10. Gunaratne DA, Barham HP, Christensen JM, Bhatia DDS, Stamm AC, Harvey RJ. Topical concentrated epinephrine (1:1000) does not cause acute cardiovascular changes during endoscopic sinus surgery. Int Forum Allergy Rhinol. 2016;6(2):135-9.

11. Yang JJ, Wang QP, Wang TY, Sun J, Wang ZY, Zuo D, et al. Marked hypotension induced by adrenaline contained in local anesthetic. Laryngoscope. 2005;115(2):348-52. 
12. Schwalm JD, Hamstra J, Mulji A, Velianou JL. Cardiogenic shock following nasal septoplasty: a case report and review of the literature. Can J Anaesth. 2008 Jun; 55(6):376-9.

13. Missvage AE, Bush RL, Kien ND, Reilly DA. The Effect of clysed and topical epinephrine on intraoperative catecholamine levels. J Trauma. 1998;45:1074-1078

14. Van Hasselt CA, Low JM, Waldron J, Gibb AG, Oh TE. Plasma catecholamine levels following topical application versus infiltration of adrenaline for nasal surgery. Anaesth Intensive Care. 1992;20(3):332-333.

15. Gessler EM, Hart AK, Dunvley TM, Greinwad JH Jr. Optimal concentration of epinephrine for vasoconstriction in ear surgery, Laryngoscope. 2001;111(10):1687-90.

16. Dunvley TM. Optimal concentration of epinephrine for vasoconstriction in neck surgery. Laryngoscope. 1996;106(11):1412-4.

17. Sarmento JKM, Tomita S, Kos AO. Topical use of adrenaline in different concentrations for endoscopic sinus surgery. Braz J Otorhinolarnyol. 2009;75(2):280-9.

18. Miller JM, Laurikainen EA, Grénman RA, Suonpää, Bredberg G. Epinephrine-induced changes in human cochlear blood flow. Am J Otol. 1994;15(3)299-305.

19. Jang CH, Cho YB, Lee JS, Kim GH, Jung WK, Pak SC. The effect of propofol infusion with topical epinephrine on cochlear blood flow and hearing: An experimental study. Int J Pediatr Otorhinolaryngol. 2016;91:23-26.

20. Choi $\mathrm{CH}$, Pak SC, Jang $\mathrm{CH}$. Effect of local anesthesia on facial nerve blood flow and muscle action potential. Int J Peditr Otorhinolaryngol. 2016;82:43-6. 
Tables

Table 1: Patient characteristics

Demographics

\begin{tabular}{ll}
\hline Age in years (range) & $42(3-83)$ \\
Female : male & $39(43 \%): 51(57 \%)$ \\
Left : right & $49(54 \%): 41(46 \%)$
\end{tabular}

Relevant Comorbidities

\begin{tabular}{lc}
\hline Cardiovascular disease & 23 \\
Endocrine disease & 16 \\
Lung disease & 5 \\
Coagulopathy & 1
\end{tabular}

Cardiovascular medication

Antiaggregation

4

Anticoagulation

1

Betablocker

8

Diuretics

7

ACE-Inhibitor

7

Sartane

2

Calcium antagonist

4

Statin

7 


\section{Surgical procedures}

\begin{tabular}{ll}
\hline Tympanoplasty Type I & $39(43,3 \%)$ \\
Stapedotomy & $25(27,8 \%)$ \\
Cholesteatoma removal & $13(14,4 \%)$ \\
Ossiculoplasty & $6(6,7 \%)$ \\
Explorative tympanotomy & $4(4,4 \%)$ \\
Tumor removal & $3(3,4 \%)$
\end{tabular}

Table 2: Intraoperative and postoperative complications

\begin{tabular}{lcc} 
Complication & Intraoperative & Postoperative \\
\hline Facial palsy & 0 & 0 \\
Sensorineural hearing loss & 0 & 1 \\
Cardiovascular events & 0 & 0 \\
High blood pressure & 2 & 0 \\
Bleeding & 0 & 2
\end{tabular}



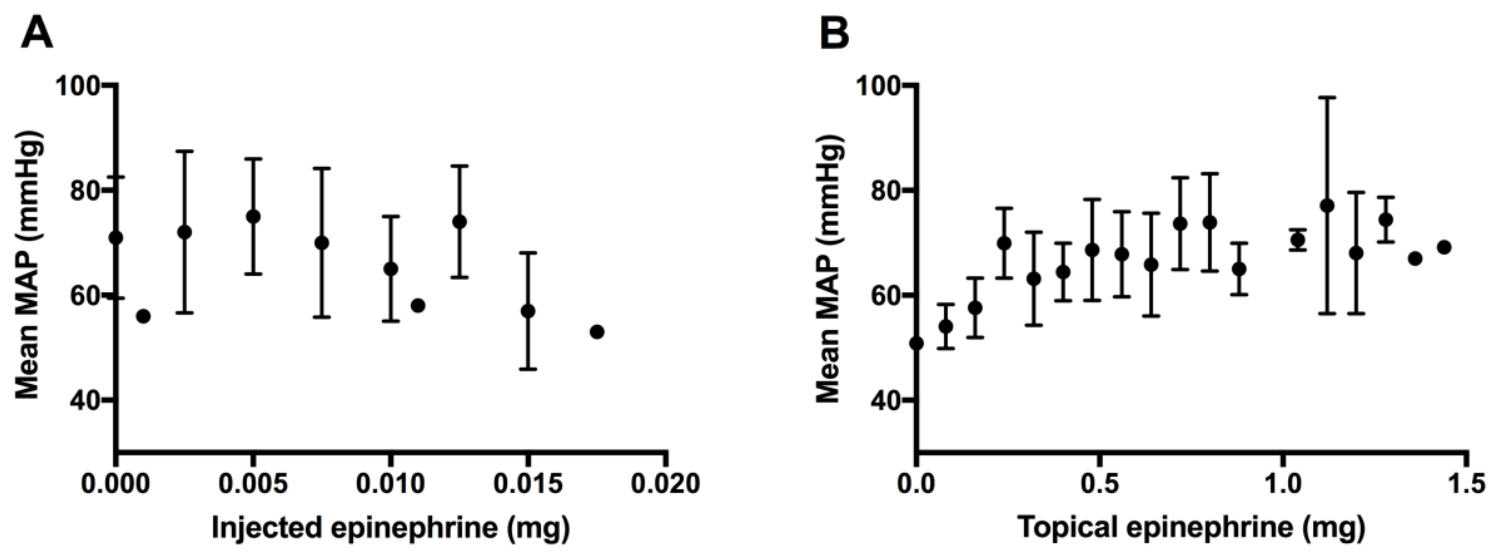

Fig. 1. Peak mean arterial pressure (MAP, $\mathrm{mmHg}$ ) after injection (Panel $\mathrm{A})$ or topical application (Panel B) of epinephrine. Values are represented as mean +/- standard deviation

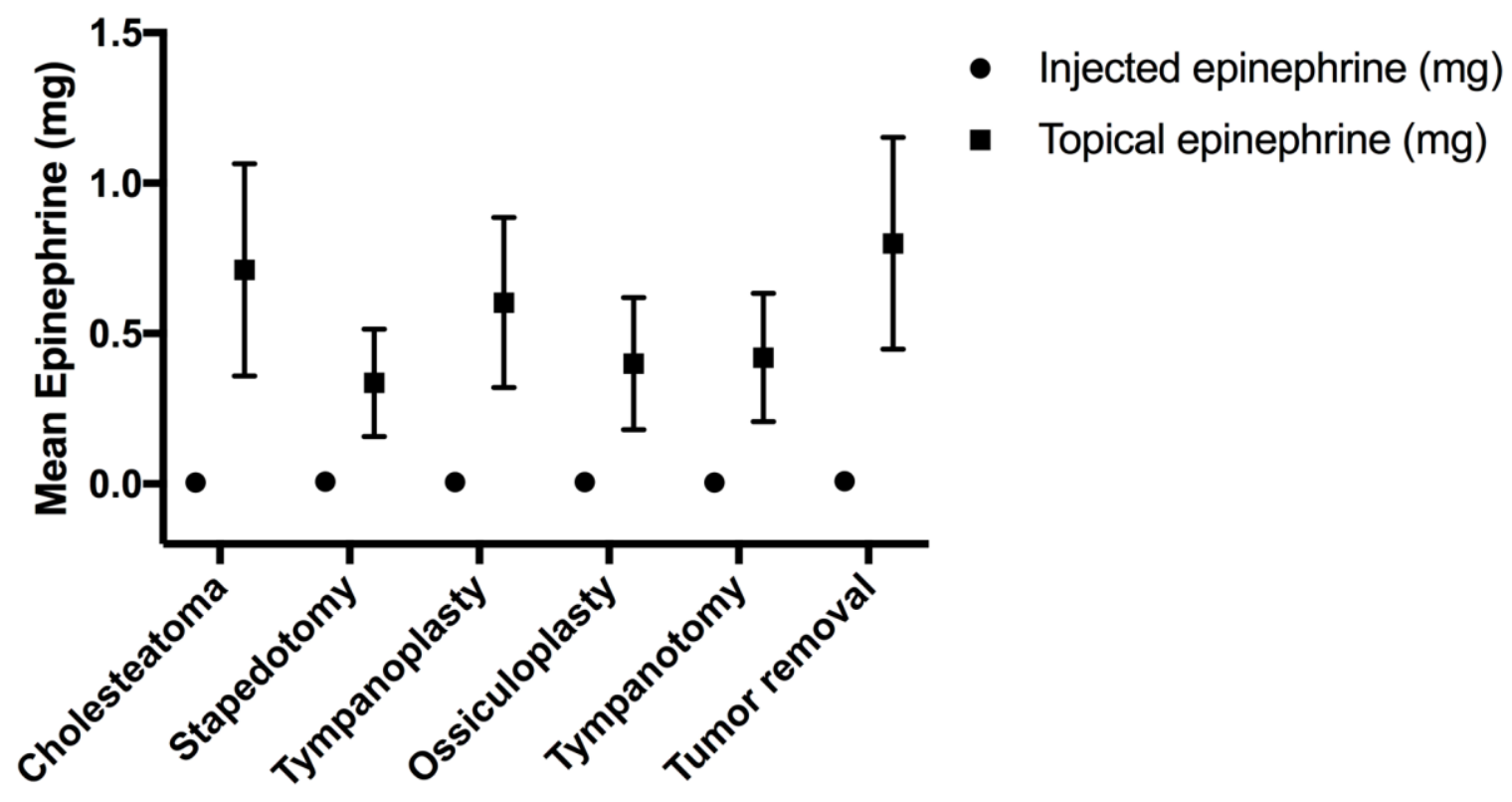

Fig. 2. Amount of injected and topical epinephrine depending on type of performed surgery. Values are represented as mean $+/$ - standard deviation 